POS PROCEEDINGS

\title{
Dynamical twisted mass fermions
}

\author{
Federico Farchioni*, Peter Hofmann, Gernot Münster \\ Institut für Theoretische Physik, Universität Münster, Wilhelm-Klemm-Str. 9, 48149 Münster, \\ Germany \\ E-mail: farchion, munsteg@uni-muenster.de, hofmann@muenster.de
}

Karl Jansen, Mauro Papinutto, Andrea Shindler, Urs Wenger*, Ines Wetzorke*

NIC, Platanenallee 6, 15738 Zeuthen, Germany

E-mail: karl.jansen, mauro.papinutto, andrea.shindler, urs.wenger, ines.wetzorke@desy.de

\section{Istvan Montvay, Enno E. Scholz, Naoya Ukita* \\ DESY, Notkestr. 85, 22607 Hamburg, Germany \\ E-mail: istvan.montvay, enno.e.scholz, naoya.ukita@desy.de \\ Luigi Scorzato \\ Institut für Physik, Humboldt-Universität zu Berlin, Newtonstr. 15, 12489 Berlin, Germany \\ E-mail: scorzato@physik.hu-berlin.de}

\section{Carsten Urbach}

NIC, Zeuthen and Institut für Theoretische Physik, Freie Universität Berlin, Arnimallee 14, 14195 Berlin, Germany

E-mail: urbach@physik.fu-berlin.de

We summarize four contributions about dynamical twisted mass fermions. The resulting report covers results for $N_{f}=2$ obtained from three different gauge actions, namely the standard Wilson plaquette gauge action [1], the DBW2 [2] and the tree-level Symanzik improved [3] gauge action. In addition, first results for $N_{f}=2+1+1$ flavours of twisted mass fermions are discussed.

XXIIIrd International Symposium on Lattice Field Theory

25-30 July 2005

Trinity College, Dublin, Ireland

\footnotetext{
* Speaker.
} 


\section{Introduction}

Within the framework of Symanzik's improvement programme $[4,5]$ the emphasis in lattice QCD has been on the construction and testing of fermion actions to avoid $O(a)$ lattice spacing artefacts. Since the lattice artefacts induced by the gauge action start with $O\left(a^{2}\right)$, the need of also exploring different gauge actions has not been considered as equally urgent. However, although this point of view is certainly valid in the asymptotic regime of small lattice spacing $a$, the situation is different when values of the lattice spacing, say, $a>0.1 \mathrm{fm}$ are considered. In this region higher order lattice artefacts my play an important role.

In the case of Wilson fermions the explicit breaking of chiral symmetry leads [6] to the appearance of a strong first order phase transition that severely affects the numerical simulations [7, 8]. As we will demonstrate in this contribution, the choice of the gauge action can have a strong effect on the strength of this phase transition and therefore it becomes important to consider both, the fermion and the gauge action to find a suitable lattice QCD action for simulations of dynamical quarks.

The twisted mass fermions approach [9] provides, among other advantages, the ideal framework for the investigation of the zero-temperature phase diagram of lattice QCD with Wilson fermions, see refs. $[10,11,12]$ for reviews on twisted mass fermions in present and past conferences.

Our present understanding can be summarized as follows, see also fig. 1. For values of the lattice spacing much coarser than $a=0.15 \mathrm{fm}$ an unphysical phase appears for small quark masses, the Aoki phase $[13,14,15]$. The transition from the standard lattice QCD phase to the Aoki phase is of second order: here the charged pions become massless. The Aoki phase only extends in the untwisted quark mass direction being absent for non vanishing twisted quark mass $\mu$. For smaller values of the lattice spacing a first order phase transition appears [7, 8, 16, 17], this time extending in the twisted mass direction $\mu$. The phase transition occurs at vanishing (untwisted) quark mass and separates the phases with opposite signs of the quark mass. This first order phase transition is reminiscent of the continuum phase transition when the quark mass is changed from positive to negative values with the corresponding jump of the scalar condensate as the order parameter of spontaneous chiral symmetry breaking. The generic phase structure of lattice QCD was discussed in refs. $[7,8,16]$.

The appearance of the first order phase transition has serious consequences, since in such a scenario the pion mass $m_{\pi}$ cannot be made arbitrarily small but assumes a minimal value, $m_{\pi}^{\min }$, which may be about $500 \mathrm{MeV}$ and hence it becomes impossible to work close to the physical value of the pion mass. It therefore becomes important to understand the phase structure of lattice QCD as a pre-requisite before starting large scale simulations. Our collaboration has performed a detailed study of the phase diagram of lattice QCD. We used Wilson fermions with and without twisted mass parameter and studied the phase diagram as a function of the lattice spacing for the Wilson plaquette, the DBW2 and the tree-level Symanzik improved gauge actions. As we will show in this contribution, the strength of the first order phase transition, namely the size of $m_{\pi}^{\min }$, depends strongly on the choice of the gauge action when comparable physical situations are tested. 


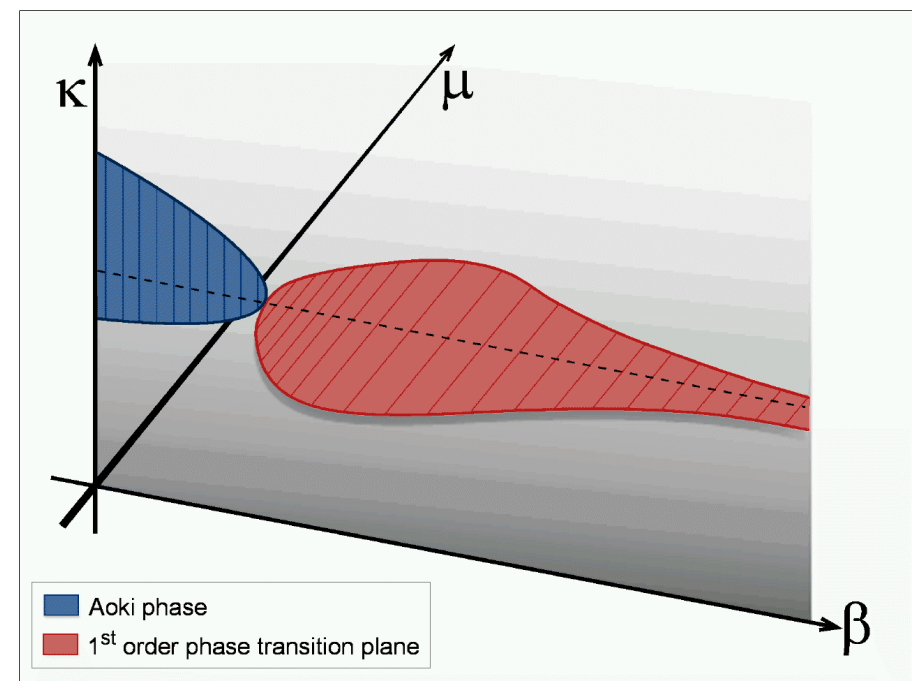

Figure 1: Current knowledge of the lattice QCD zero-temperature phase diagram with Wilson fermions as a function of the inverse gauge coupling $\beta \propto 1 / g^{2}$, the hopping parameter $\kappa$ and the twisted mass parameter $\mu$.

Since lattice chiral perturbation theory $(\chi \mathrm{PT})[6,18,19,20,21,22]$ predicts a weakening of the first order phase transition toward the continuum limit, it is interesting to check this prediction and, in particular, to investigate how fast the transition weakens when the continuum limit is approached. The answer to the latter question will naturally depend on the choice of the actions that are used for the gauge and the fermion fields. Moreover, the predictions of $\chi \mathrm{PT}$ for e.g. the quark mass dependence of the pion mass and the pseudoscalar decay constant at such a phase transition can be directly confronted to results from numerical simulations.

This contribution is organized as follows. In section 2 we will give the definitions of the actions we will use. In sections 3, 4 and 5 we will give our results for the Wilson plaquette, the DBW2 and the tree-level Symanzik improved actions, respectively. In section 6 we will confront our lattice data with lattice $\chi$ PT. In section 7 we will provide first results from simulations with $N_{f}=2+1+1$ flavors of quarks. Finally, we will conclude in section 8 .

\section{Lattice action}

The lattice action for a doublet of degenerate twisted mass Wilson fermions (in the so-called "twisted basis") reads

$$
S_{q}=\sum_{x}\left\{\left(\bar{\chi}_{x}\left[\mu_{\kappa}+i \gamma_{5} \tau_{3} a \mu\right] \chi_{x}\right)-\frac{1}{2} \sum_{\mu= \pm 1}^{ \pm 4}\left(\bar{\chi}_{x+\hat{\mu}} U_{x \mu}\left[r+\gamma_{\mu}\right] \chi_{x}\right)\right\},
$$

with $\mu_{\kappa} \equiv a m_{0}+4 r=1 / 2 \kappa, r$ the Wilson-parameter, set in our simulations to $r=1, a m_{0}$ the bare "untwisted" quark mass in lattice units ( $\kappa$ is the conventional hopping parameter) and $\mu$ the twisted quark mass; we also define $U_{x,-\mu}=U_{x-\hat{\mu}, \mu}^{\dagger}$ and $\gamma_{-\mu}=-\gamma_{\mu}$. 
For the gauge sector we consider the one-parameter family of actions including planar rectangular $(1 \times 2)$ Wilson loops $\left(U_{x \mu \nu}^{1 \times 2}\right)$ :

$$
S_{g}=\beta \sum_{x}\left(c_{0} \sum_{\mu<v ; \mu, v=1}^{4}\left\{1-\frac{1}{3} \operatorname{Re} U_{x \mu \nu}^{1 \times 1}\right\}+c_{1} \sum_{\mu \neq v ; \mu, v=1}^{4}\left\{1-\frac{1}{3} \operatorname{Re} U_{x \mu \nu}^{1 \times 2}\right\}\right),
$$

with the normalization condition $c_{0}=1-8 c_{1}$. We consider in this contribution the three cases: i.) Wilson plaquette gauge action, $c_{1}=0$, ii.) DBW2 gauge action [23], $c_{1}=-1.4088$, iii.) tree-level Symanzik improved gauge action (tlSym) [24], $c_{1}=-1 / 12$.

\section{Wilson plaquette gauge action}

The first action we investigate here is the Wilson plaquette gauge action. We studied the lattice spacing dependence of several quantities [17] keeping the twisted mass and the lattice size roughly fixed to $r_{0} \mu \approx 0.03$ ( $r_{0}$ being the Sommer parameter [25], we assume $r_{0}=0.5 \mathrm{fm}$ throughout this contribution) and $L \approx 2 \mathrm{fm}$. We varied $\beta=6 / g^{2}$ in the range $\beta=5.1-5.3$. For each value of the hopping parameter we performed a hot and a cold start in order to check for co-existing values of physical observables which we have chosen as the pion mass $m_{\pi}$ and the rho meson mass $m_{\rho}$, extracted from the usual correlation functions. Another quantity is the amplitude $f_{\chi}^{P S}$, which reduces to the pion decay constant $f_{\pi}$ for $\mu=0$, defined as

$$
f_{\chi}^{P S}=\frac{r_{A P}}{m_{\pi}}\langle 0|P(0)| \pi\rangle \equiv \frac{r_{A P}}{m_{\pi}} g_{\pi}, \quad \text { where } \quad r_{A P}=\frac{\left\langle 0\left|A_{0}(0)\right| \pi\right\rangle}{\langle 0|P(0)| \pi\rangle}
$$

is extracted from the asymptotic behavior of the ratio of the axialvector-pseudoscalar correlator $C_{A P}\left(x_{0}\right)$ to the pseudoscalar-pseudoscalar correlator $C_{P P}\left(x_{0}\right)$ (cf. ref [17] and references therein for details): $\frac{C_{A P}\left(x_{0}\right)}{C_{P P}\left(x_{0}\right)}=r_{A P} \tanh \left[m_{\pi}\left(T / 2-x_{0}\right)\right]$. Moreover we measure the untwisted PCAC quark $\operatorname{mass} m_{\chi}^{P C A C}$ :

$$
m_{\chi}^{P C A C}=\frac{f_{\chi}^{P S} m_{\pi}^{2}}{2\langle 0|P(0)| \pi\rangle} \equiv \frac{f_{\chi}^{P S} m_{\pi}^{2}}{2 g_{\pi}}
$$

Note that the physical decay constant (PCAC quark mass) is obtained by a combination of $f_{\chi}^{P S}$ and the twist angle $\omega\left(m_{\chi}^{P C A C}\right.$ and the twisted mass $\left.\mu\right)$, see also section 4 .
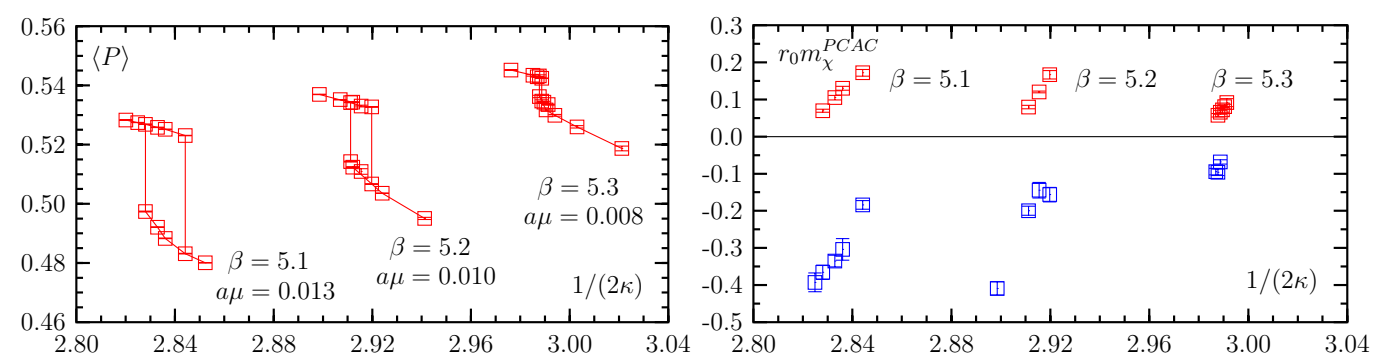

Figure 2: Lattice spacing dependence of the plaquette expectation value (left) and the PCAC quark mass (right). 
The plaquette expectation value shows the typical behavior at a first order phase transition with meta-stable branches, illustrated in fig. 2 (left). For the PCAC quark mass the two branches correspond to positive and negative quark mass, respectively (see fig. 2 (right)). In both cases the gap decreases from $\beta=5.1$ to 5.3 and the meta-stability region in $1 / 2 \kappa$ shrinks. For the pion mass shown in fig. 3 (left) this fact has the important consequence that only a minimal pion mass $\sim 500$ $\mathrm{MeV}$ can be reached. If one attempts to lower the mass further by tuning $\mu$ or $\kappa$, a jump to the other phase occurs. In addition, the scale parameter $r_{0} / a$ is quite different in the two phases and its mass dependence is non-negligible, as illustrated in fig. 3 (right).
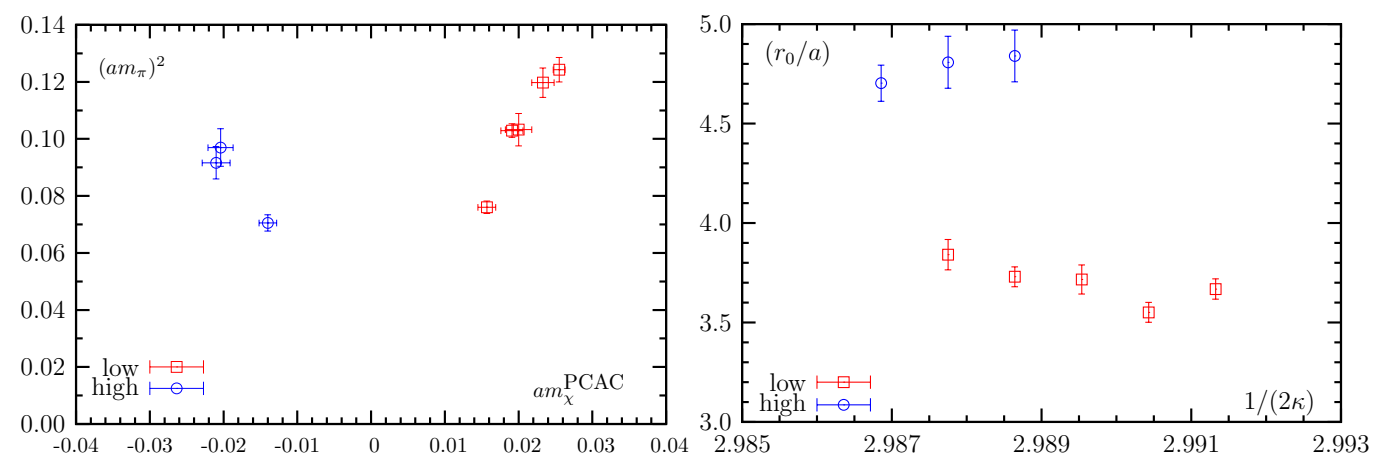

Figure 3: Pion mass vs. PCAC quark mass (left) and mass dependence of $r_{0} / a$ (right) at $\beta=5.3$. "Low" and "high" refer to the low plaquette (positive quark mass) and the high plaquette (negative quark mass) phase, respectively.

In the light of these observations the interesting question arises at which $\beta$-value or lattice spacing we can safely simulate pions with masses as light as $\sim 300 \mathrm{MeV}$ in order to make contact with $\chi$ PT. With the present data it is very difficult to make an extrapolation, since there is a large ambiguity in the determination of the minimal pion mass, the lever arm is very short due to the small range of $\beta=5.1-5.3$ covered and furthermore due to the large difference of the scale parameter $r_{0} / a$ in the two phases. Nevertheless, a qualitative estimate would be a lattice spacing of 0.07-0.1 fm, where one should be able to reach small pion masses without being affected by the first order phase transition. This makes even $L=2 \mathrm{fm}$ simulations, e.g. for a detailed scaling study, very demanding. Therefore alternative gauge actions that lead to a reduced strength of the first order phase transition are investigated in the next sections.

\subsection{Scaling behavior}

Here we study the scaling behavior with our current data for the Wilson plaquette action, also including the data from the DBW2 gauge action, see sec. 4. To this end we express the physical quantities in dimensionless variables. We first define a reference quark mass by $\left(r_{0} m_{\pi}\right)^{2}=1.5$. At this reference quark mass, we then determine* reference values for the quantities we are interested in, i.e. $r_{0} / a, m_{\chi}^{P C A C}, m_{\pi}, m_{\rho}$ and $f_{\chi}^{P S}$. This allows to define dimensionless ratios such as for the quark mass, $\sigma=\frac{m_{\chi}^{P C A C}}{\left.m_{\chi}^{P A C}\right|_{\text {ref }}}$ or other observables, $R_{O}=\frac{O}{\left.O\right|_{\text {ref }}}$. The so determined observables, measured in units of their reference values, are universal and can be compared at different $\beta$-values and for different actions.

\footnotetext{
* We consider only the positive quark mass phase which corresponds to standard lattice QCD.
} 


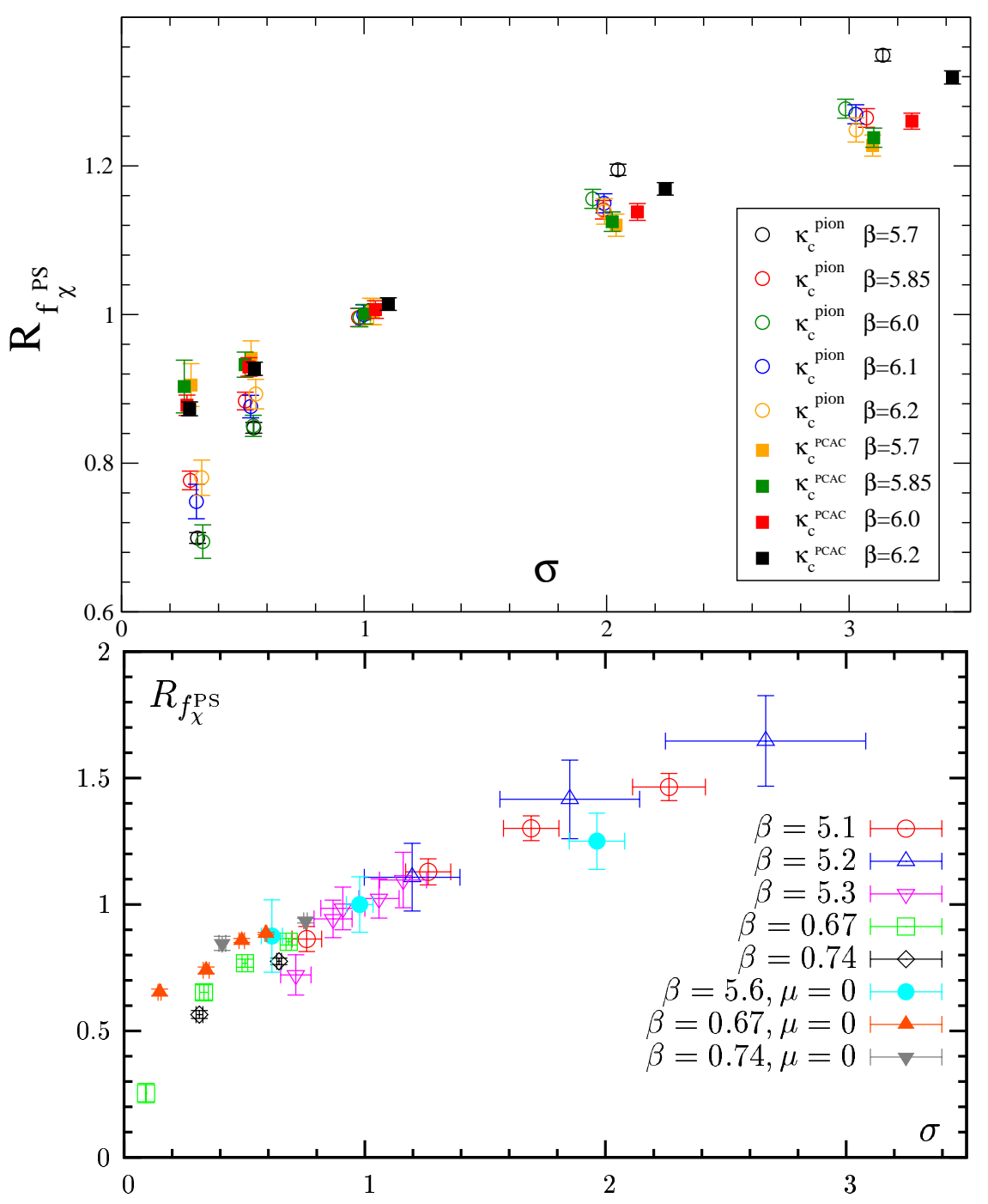

Figure 4: Scaling ratios $R_{f_{\chi}^{P S}}$ in the quenched approximation (upper panel) and for dynamical fermions (lower panel). The lower panel also includes results from the DBW2 action at $\beta=0.67$ and $\beta=0.74$ (see also sec. 4).

In fig. 4 (lower panel) we show as an example the scaling ratio for our dynamical fermion data of the Wilson plaquette and the DBW2 gauge actions. We observe no scaling violations for the data from the Wilson plaquette gauge action at $\beta=5.1-5.3$ within the (large) statistical errors. Moreover, we see agreement with data at $\beta=5.6$ for pure Wilson fermions without twisted mass, produced for the algorithmic study of an HMC variant [26, 27]. For the data from the DBW2 gauge action at smaller masses we observe instead a slight difference in the scaling behavior of the $\mu=0$ and $\mu \neq 0$ results. This might indicate that such a difference could be detected for the Wilson plaquette gauge action only at higher beta values, where smaller quark masses can be reached. Similar plots were also obtained for the scaling ratios $R_{m_{\pi}^{2}}$ and $m_{\pi} / m_{\rho}$. For comparison, we also show in fig. 4 (upper panel) an example of the ratio $R_{f_{\chi} P S}$ for quenched data at full twist, $\kappa=\kappa_{c}$ [28]. 
Here we observe scaling violations for our coarsest lattice at $\beta=5.7$ and a difference in the scaling behavior at small masses for different discretizations of twisted mass fermions, realized by using two definitions of the critical mass ( $\kappa_{c}^{\text {pion }}$ and $\kappa_{c}^{P C A C}$, see ref. [28]). This might provide a warning that at small values of the quark mass the lattice artefacts could be significant and that scaling violations might show up.

\section{DBW2 action: analysis of twist angle and physical quantities}

In this section we analyze several interesting quantities in the context of simulations of $N_{f}=2$ flavors of twisted mass fermions with the DBW2 gauge action. This particular setup was studied in [16] on a $12^{3} \cdot 24$ lattice at $\beta=0.67$ with spatial extension $L \simeq 2 \mathrm{fm}$, lattice spacing $a \simeq 0.18 \mathrm{fm}$, $r_{0} \mu \simeq 0.03$ : the minimal pion mass found here was $m_{\pi} \simeq 360 \mathrm{MeV}$. Signs of metastabilities are hardly detectable in this situation, which is a clear improvement compared to the Wilson plaquette gauge action, cf. sec. 3 . In this section we also consider data from $16^{3} \cdot 32$ lattices at a finer value of the lattice spacing, $\beta=0.74$, keeping approximately the same volume and value of the twisted quark mass as in the aforementioned simulations.

\subsection{Twist angle}

The twist angle $\omega$ defines the chiral rotation relating twisted mass QCD to ordinary QCD. In the case of the chiral currents the rotation reads (considering only charged currents, $a=1,2$ ):

$$
\begin{aligned}
& \hat{V}_{x \mu}^{a}=Z_{V} V_{x \mu}^{a} \cos \omega+\varepsilon_{a b} Z_{A} A_{x \mu}^{b} \sin \omega, \\
& \hat{A}_{x \mu}^{a}=Z_{A} A_{x \mu}^{a} \cos \omega+\varepsilon_{a b} Z_{V} V_{x \mu}^{b} \sin \omega
\end{aligned}
$$

where the hatted currents on the 1.h.s. denote the chiral currents of QCD (physical currents), while the currents on the r.h.s. are the corresponding bilinears of the quark-field in the twisted $(\chi-)$ basis. Note that the renormalization constants of these bilinears, $Z_{V}$ and $Z_{A}$, are involved. For a given choice of the lattice parameters, the twist angle $\omega$ is determined by requiring parity conservation for matrix elements of the physical currents $[8,16]$. Since unknown renormalization constants are involved, two conditions are required, our choice being:

$$
\begin{aligned}
& \sum_{\vec{x}}\left\langle\hat{V}_{x 0}^{+} P_{y}^{-}\right\rangle=0, \\
& \sum_{\vec{x}, i}\left\langle\hat{A}_{x i}^{+} \hat{V}_{y i}^{-}\right\rangle=0 .
\end{aligned}
$$

The solution of eqs. (4.3) and (4.4) with eqs. (4.1) and (4.2) gives a direct determination of the twist angle $\omega$ and of the ratio $Z_{A} / Z_{V}$ from lattice data, see [16] for details. Eq. (4.3) implies e.g.

$$
\cot \omega_{V} \equiv \frac{Z_{V}}{Z_{A}} \cot \omega=-i \frac{\sum_{\vec{x}}\left\langle A_{x 0}^{+} P_{y}^{-}\right\rangle}{\sum_{\vec{x}}\left\langle V_{x 0}^{+} P_{y}^{-}\right\rangle} .
$$

In particular at full twist where $\omega=\pi / 2$ the condition reads $\sum_{\vec{x}}\left\langle A_{x 0}^{+} P_{y}^{-}\right\rangle=0$ or $\cot \omega_{V}=0$. The full twist situation can be also obtained by requiring the vanishing of the PCAC quark mass $m_{\chi}^{P C A C}$. 
One can easily see that using the PCAC quark mass as a criterion for full twist just amounts to a different definition of the twist angle: the current is replaced by its divergence in (4.3). So the two criteria are equivalent and differ (at most) by $O(a)$ effects. The numerical equivalence of the two criteria for full twist is illustrated by fig. 5 .
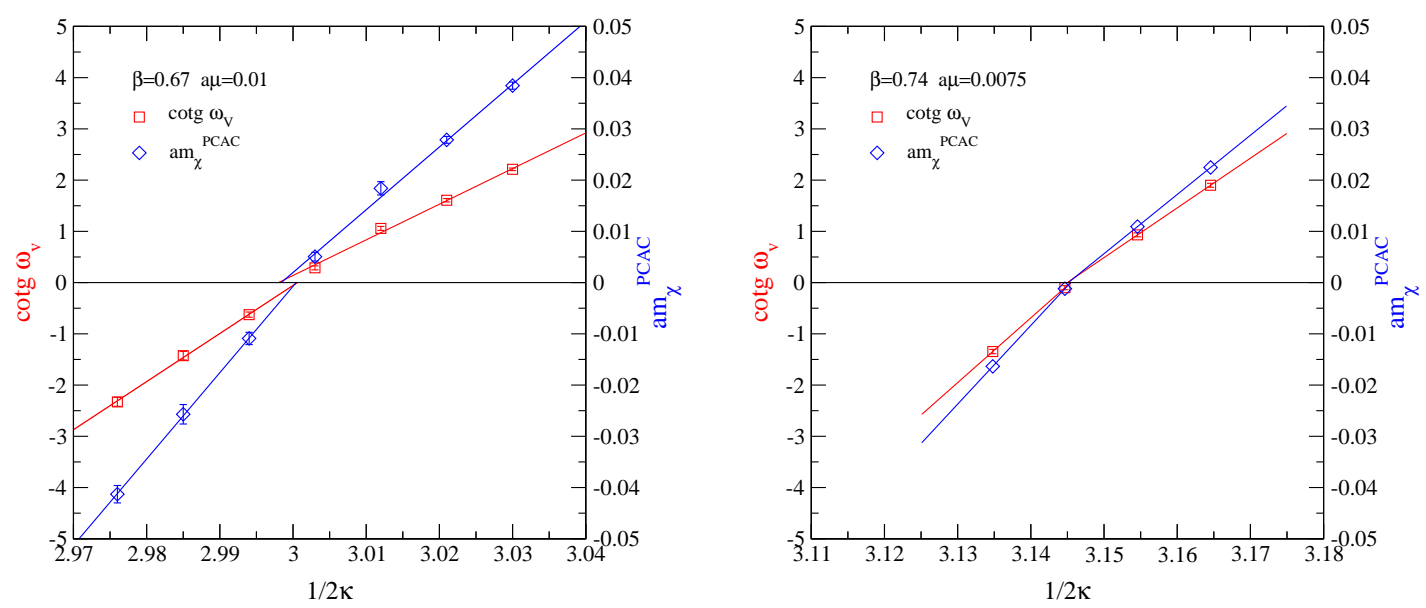

Figure 5: Determination of the critical hopping parameter $\kappa_{c}$ corresponding to full twist by parity-restoration and by extrapolating to zero the untwisted PCAC quark mass $m_{\chi}^{P C A C}$. The small discrepancy observed at $\beta=0.67$ (left panel) between extrapolations from positive and negative quark masses is probably a residual effect of the first order phase transition (crossover). For $\beta=0.74$ (right panel), extrapolations from both sides give consistent results.

\subsection{Physical quark mass and pion decay constant}

The knowledge of the twist angle is necessary for the determination of physical quantities like the quark mass and the pion decay constant. The physical PCAC quark mass $m_{q}^{P C A C}$ can be obtained from the Ward identity for the physical axialvector current:

$$
\left\langle\nabla_{\mu}^{*} \hat{A}_{x \mu}^{+} P_{y}^{-}\right\rangle=2 m_{q}^{P C A C}\left\langle P_{x}^{+} P_{y}^{-}\right\rangle .
$$

From eqs. (4.1) and (4.2) follows

$$
\hat{A}_{x \mu}^{a}=\hat{V}_{x \mu}^{a} \cot \omega+\varepsilon_{a b} \tilde{V}_{x \mu}^{b}(\sin \omega)^{-1}
$$

where the conserved vector current of the $\chi$-fields $\tilde{V}_{x \mu}^{b}$ has been now considered, for which $Z_{V}=1$. Inserting the above result in the Ward identity (4.6) and using parity restoration for the physical currents one obtains:

$$
m_{q}^{P C A C}=-i(2 \sin \omega)^{-1} \frac{\left\langle\nabla_{\mu}^{*} \tilde{V}_{x \mu}^{+} P_{y}^{-}\right\rangle}{\left\langle P_{x}^{+} P_{y}^{-}\right\rangle}=Z_{A}(\cos \omega)^{-1} m_{\chi}^{P C A C} .
$$

Analogously, for the physical pion decay constant $f_{\pi}$ we use

$$
f_{\pi}=m_{\pi}^{-1}\left\langle 0\left|\hat{A}_{0}^{+}(0)\right| \pi^{+}\right\rangle=-i\left(m_{\pi} \sin \omega\right)^{-1}\left\langle 0\left|\tilde{V}_{0}^{+}(0)\right| \pi^{+}\right\rangle .
$$


Table 1: Chiral extrapolation $\left(m_{q}^{P C A C}=0\right)$ of the Sommer scale parameter $r_{0}$ and pion decay constant $f_{\pi}$; the scale independent combination $f_{\pi} r_{0}$ is also reported. Only data with positive twisted quark masses have been used for the extrapolations, with the exception of the point near full twist at $\mu=7.5 \cdot 10^{-3}$.

\begin{tabular}{cccccc}
\hline \hline$\beta$ & $\mu$ & \multicolumn{1}{c}{$r_{0} / a$} & $a(\mathrm{fm})$ & $a f_{\pi}$ & $f_{\pi} r_{0}$ \\
\hline \hline 0.67 & $1.0 \cdot 10^{-2}$ & $2.680(68)$ & $0.1866(72)$ & $0.1171(59)$ & $0.320(10)$ \\
0.74 & $7.5 \cdot 10^{-3}$ & $4.11(13)$ & $0.1216(39)$ & $0.0726(25)$ & $0.309(15)$ \\
\hline \hline
\end{tabular}

In fig. 6 the pion decay constant is plotted as a function of the quark mass (the simulation points for negative quark masses are not included). The figures also show the determination of $f_{\pi}$ by the axialvector current $A_{x \mu}^{a}$ : a formula similar to eq. (4.9) applies in this case, where however the factor $1 / \sin \omega$ is replaced by $1 / \cos \omega$. In the interesting region near full twist this factor introduces the large fluctuations in the estimate of $f_{\pi}$ observed in the data. Moreover in the case of $A_{x \mu}^{a}$ the decay constant has not yet the right normalization of the continuum: a $Z_{A}$ factor is missing. In the case of the conserved vector current on the contrary the lattice determination of $f_{\pi}$ has automatically the correct normalization [29, 30]. If we exclude the lightest point at $\beta=0.67$, which is likely to be under the influence of residual metastability effects, $f_{\pi}$ seems to be characterized by a linear dependence upon the quark mass. On the basis of this observation we try a simple linear extrapolation to the chiral limit $m_{q}^{P C A C}=0$, see table 1 for the numerical results. (Of course deviations from this linear behavior could be present for lighter quark masses, where chiral logarithms might play a role.) We see that the two $\beta$-values give compatible values for $f_{\pi} r_{0}$, suggesting that there are no large scaling violations for this action. These values, obtained with $N_{f}=2$, are also near to the physical value $f_{\pi} r_{0}=0.33$.
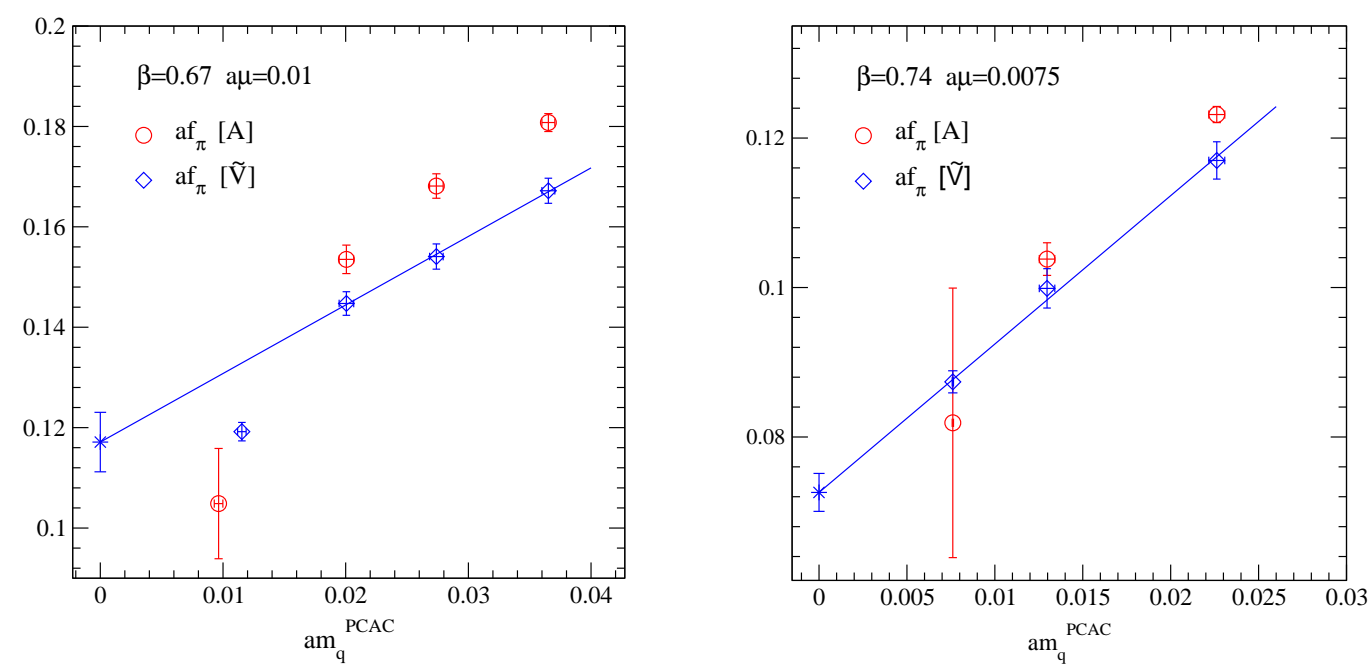

Figure 6: The pion decay constant $f_{\pi}$ as a function of the PCAC quark mass $m_{q}^{P C A C}$. 
Table 2: The renormalization constants $Z_{V}, Z_{A}$ at full twist (extrapolating from positive quark masses) with comparison to 1-loop perturbative estimates (PT) and tadpoleimproved perturbative estimates (TI)[32].

\begin{tabular}{ccclcc}
\hline \hline$\beta$ & $\mu$ & Op. & \multicolumn{1}{c}{$Z$} & $Z(\mathrm{PT})$ & \multicolumn{1}{c}{$Z(\mathrm{TI})$} \\
\hline \hline 0.67 & $1.0 \cdot 10^{-2}$ & $V$ & $0.5650(11)$ & 0.6089 & 0.6531 \\
0.74 & $7.5 \cdot 10^{-3}$ & $V$ & $0.6217(23)$ & 0.6459 & 0.6892 \\
0.67 & $1.0 \cdot 10^{-2}$ & $A$ & $0.952(30)$ & 0.7219 & 0.7176 \\
0.74 & $7.5 \cdot 10^{-3}$ & $A$ & $0.944(74)$ & 0.7482 & 0.7735 \\
\hline \hline
\end{tabular}

\subsection{Renormalization constants}

The renormalization constant of the vector current $Z_{V}$ can be determined on the basis of the non-renormalization property of the conserved current $\tilde{V}_{x \mu}$ [31]. In the case of twisted mass QCD we use

$$
Z_{V}^{\text {latt }}=\frac{\left\langle 0\left|\tilde{V}_{0}^{+}\right| \pi^{+}\right\rangle}{\left\langle 0\left|V_{0}^{+}\right| \pi^{+}\right\rangle}
$$

Note that in twisted mass QCD the time-component of the vector current couples the vacuum to the pseudoscalar meson: in the most interesting region near full twist this coupling is (in our twisted basis) maximal. In lattice QCD with ordinary Wilson fermions the analogous procedure has to rely on the noisier matrix element of the vector particle. Eq. (4.10) represents a mass dependent prescription. We obtain a mass independent determination of $Z_{V}$ by extrapolating $Z_{V}^{\text {latt }}$ to full twist. In this situation the theory is $O(a)$ improved and $Z_{V}^{\text {latt }}$ has only $O\left(a^{2}\right)$ lattice deviations. (In particular residual $O\left((\mu a)^{2}\right)$ terms can be eliminated by a $\mu \rightarrow 0$ extrapolation.) The results are reported in table 2; the renormalization of the axialvector current $Z_{A}$ is also reported: this is obtained by combining the direct determination of $Z_{V}$ (4.10) and the determination of $Z_{A} / Z_{V}$ from the parity restoration (see above) after extrapolation to full twist. Observe that the conditions (4.3), (4.4) hold in general up to $O(a)$ parity violations: imposing (as we do) exact parity restoration for the considered m.e. leads to a mass dependent determination of the ratio $Z_{A} / Z_{V}$.

\section{Tree-level Symanzik improved gauge action}

In this section we will now concentrate on the tree-level Symanzik improved (tlSym) gauge action for which the coefficient $c_{1}=-1 / 12$. The reasons for the choice of this action are manifold - firstly, the action is designed to show a good and smooth behavior in the perturbative regime; secondly, in many quenched scaling studies the action has proved to behave well also in the nonperturbative regime; and thirdly, the additional coefficient $c_{1}$ is rather small in absolute value as compared to other improved actions like the DBW2. We have simulated several values of $\kappa$ close to $\kappa_{c}$, i.e. close to maximal twist, at three different values of $\beta$ using a HMC algorithm variant with mass preconditioning and multiple time scale integration [26, 27].

In order to check for a possible phase transition and corresponding metastabilities we measure the average plaquette value as a function of the hopping parameter $\kappa$ on runs with hot (disordered) 
and cold (ordered) starting configurations. Since the metastability, if any, will show up around $\kappa_{c}$ we concentrate our hot and cold runs on $\kappa$-values closest to $\kappa_{c}$ only. Fig. 7 (left) shows the results for the average plaquette value for our coarsest lattice at $\beta=3.65,12^{3} \cdot 24$ and $r_{0} \mu=0.038$. Here and in the rest of the section the red circles and blue squares represent the results from the hot and cold starts, respectively. From fig. 7 (left) it is clear that there seems to be no metastability as far as
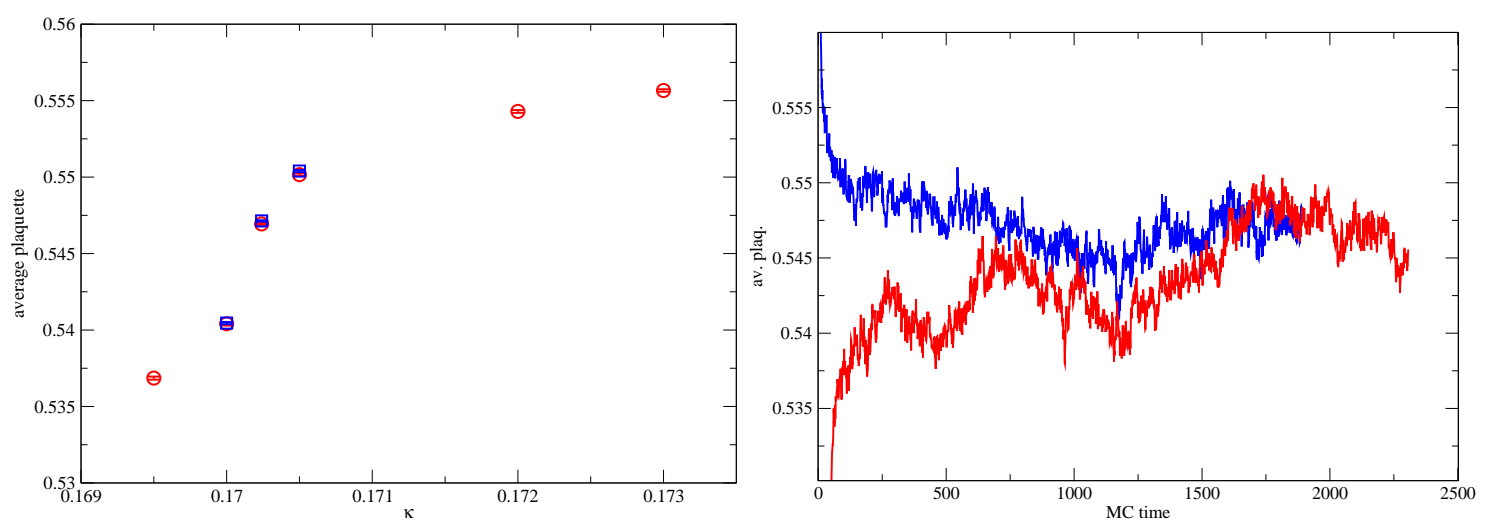

Figure 7: Left panel: Average plaquette value vs. $\kappa$ at $\beta=3.65, r_{0} \mu=0.038$ on a $12^{3} \cdot 24$ lattice from hot (red circles) and cold starts (blue squares). Right panel: Plaquette MC time history for two runs at $\beta=3.65, r_{0} \mu=0.038, \kappa=0.17024$ on a $12^{3} \cdot 24$ lattice starting from hot (red line coming from below) and cold configuration (blue line coming form above).

the plaquette value is concerned, but the existence of a phase transition at this parameter set can of course not be excluded. Indeed, the rise of the plaquette value around $\kappa_{c} \simeq 0.17025$ points toward the fact that we are at least in the vicinity of a phase transition and we conclude that a lower value of $\mu$ would hardly be possible to simulate in practice. The vicinity of the phase transition is also reflected in the MC time histories of the plaquette value. We observe a rather strong critical slowing down leading both to a very slow thermalization and large fluctuations of the plaquette value over several hundreds of trajectories. It is evident from the MC time history shown in fig. 7 (right) that any statement about the plaquette value itself as well as any error estimate are very difficult to make and should hence be taken with care. The figure also illustrates the fact that simulations close to or at $\kappa_{c}$ (i.e. at maximal twist angle) are rather difficult, at least at such large values of the lattice spacing $(a \simeq 0.13 \mathrm{fm})$ and values of $\mu$ as chosen here.

Let us now move to our simulations at $\beta=3.75$. Fig. 8 (left) shows the average plaquette value vs. $\kappa$ at $\beta=3.75, r_{0} \mu=0.020$ on a $12^{3} \cdot 24$ lattice from hot (red circles) and cold starts (blue squares). Compared to fig. 7 we are now at finer lattice spacing $\left(r_{0} / a=3.76(9)\right.$ at $\beta=3.65$ vs. $r_{0} / a=4.1(2)$ at $\beta=3.75$ ), but on the other hand also our value of the twisted mass is halved, so we do not necessarily expect the situation to be better. Indeed, we find that at the value of $\kappa=0.1660$ that is closest to $\kappa_{c}$ the average plaquette value from the hot and the cold start do not match each other, which is pointing to the existence of a phase transition and a corresponding metastability at this set of parameters. On the other hand, considering the large fluctuations of the plaquette similar to the ones in fig. 7, the runs here are presumably not long enough to allow reliable estimates and the metastability might disappear as the MC time history becomes longer. Nevertheless, the strong rise of the plaquette around $\kappa_{c}$ points toward the fact that we are at least close to a transition, even though the rise appears to be much weaker than at $\beta=3.65$. 

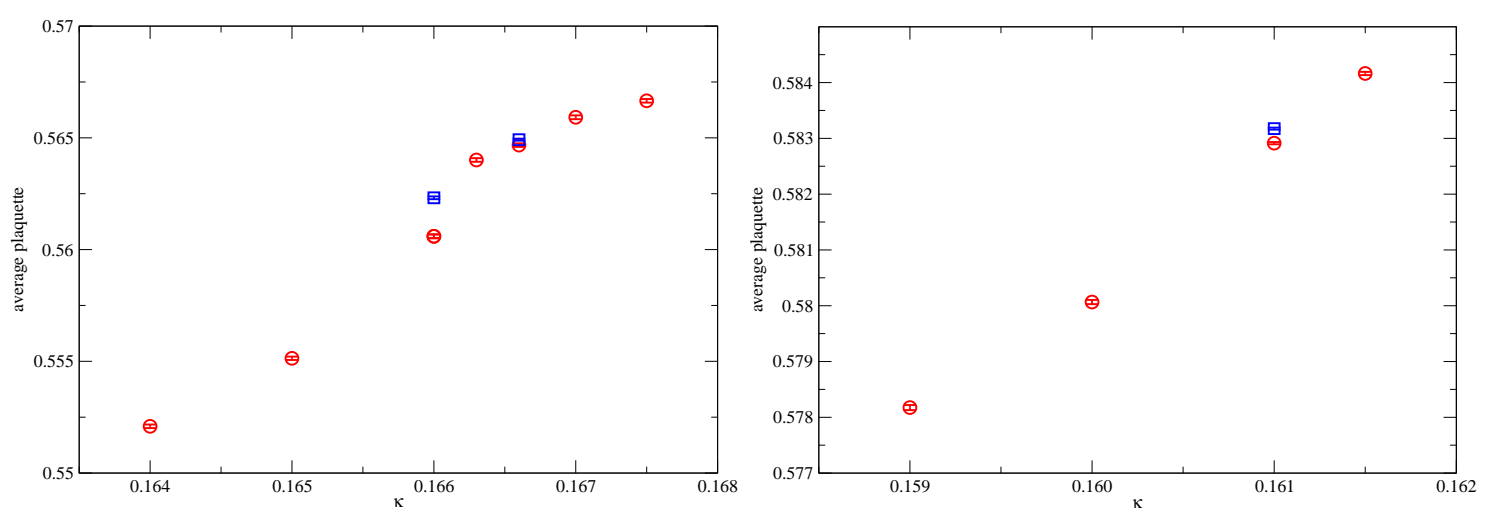

Figure 8: Left panel: Average plaquette value vs. $\kappa$ at $\beta=3.75, r_{0} \mu=0.020$ on a $12^{3} \cdot 24$ lattice from hot (red circles) and cold starts (blue squares). Right panel: Average plaquette value vs. $\kappa$ at $\beta=3.90, r_{0} \mu=$ 0.041 on a $16^{3} \cdot 32$ lattice from hot (red circles) and cold starts (blue squares).

The situation at $\beta=3.90$ is illustrated in fig. 8 (right) where $r_{0} \mu=0.041$ corresponds again to our larger value of $\mu$. Here we observe a very smooth dependence of the plaquette value on $\kappa$ and there seems to be no trace of a cross-over or even a nearby phase transition. (We attribute the fact that the plaquette value at $\kappa=0.1610 \simeq \kappa_{c}$ from the hot and cold start do not match exactly to the shortness of our runs which prevents an accurate determination of the average plaquette value.)

Next we consider the untwisted PCAC quark mass $m_{\chi}^{P C A C}$, cf. eq. (3.2), as a function of $\kappa$ for $\beta=3.75$ and 3.90 in fig. 9. For $\beta=3.75$ (left) we find that the mismatch between the plaquette
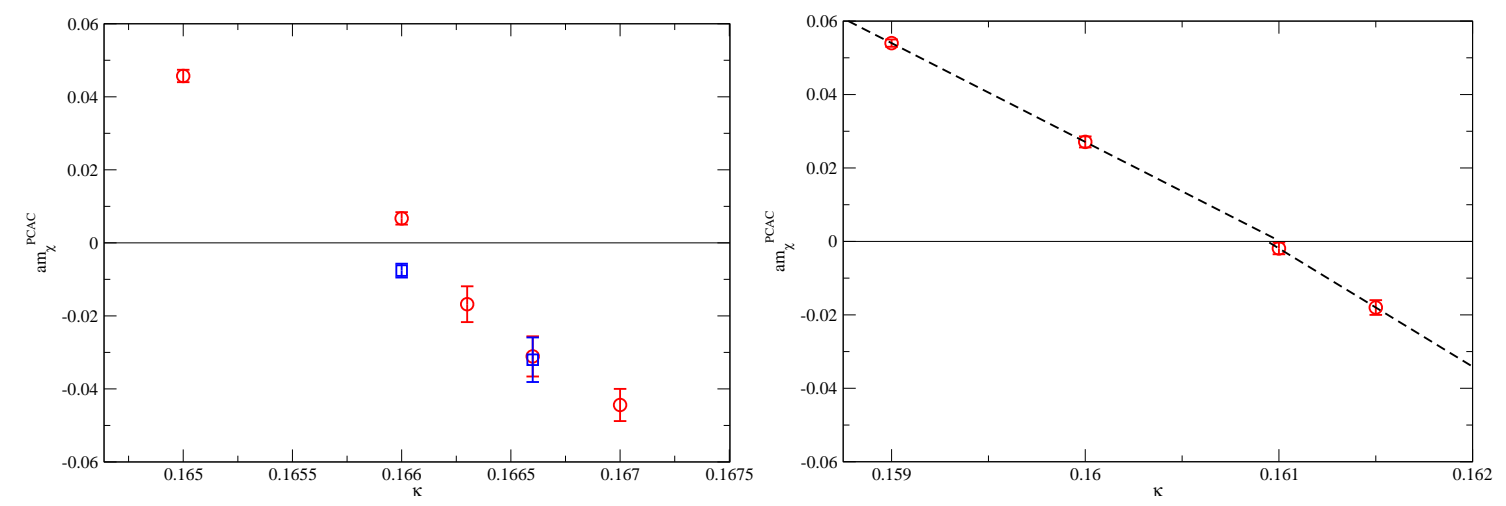

Figure 9: Untwisted PCAC quark mass $m_{\chi}^{P C A C}$ vs. $\kappa$ from hot (red circles) and cold starts (blue squares). At $\beta=3.75, r_{0} \mu=0.020$ on a $12^{3} \cdot 24$ (left plot) and $\beta=3.90, r_{0} \mu=0.041$ on a $16^{3} \cdot 32$ lattice (right plot).

value from the hot and the cold start at $\kappa=0.1660 \simeq \kappa_{c}$ is also reflected in the PCAC quark mass, although we expect that this mismatch will eventually go away for long enough runs as discussed before. Nevertheless, even then it seems unlikely that extrapolations to $m_{\chi}^{P C A C}=0$ from $\kappa$-values above and below $\kappa_{c}$ will coincide. This is in contrast to the situation at $\beta=3.90$ (right plot) where we observe a smooth dependence of $m_{\chi}^{P C A C}$ on $\kappa$ throughout a wide range of $\kappa$-values. Indeed, extrapolations to $m_{\chi}^{P C A C}=0$ from $\kappa$-values above and below $\kappa_{c}$ nicely coincide while the slopes are different (in consistency with expectations from $\chi$ PT [18, 19, 20, 21, 22]).

Let us now turn to the pion mass squared $m_{\pi}^{2}$ as a function of the PCAC quark mass $m_{\chi}^{P C A C}$ which we plot in fig. 10 for $\beta=3.75, r_{0} \mu=0.020,12^{3} \cdot 24$ on the left and for $\beta=3.90, r_{0} \mu=$ 
$0.041,16^{3} \cdot 32$ on the right. At $\beta=3.75$ the pion mass that is realized at $r_{0} \mu=0.020$ is $m_{\pi}=$
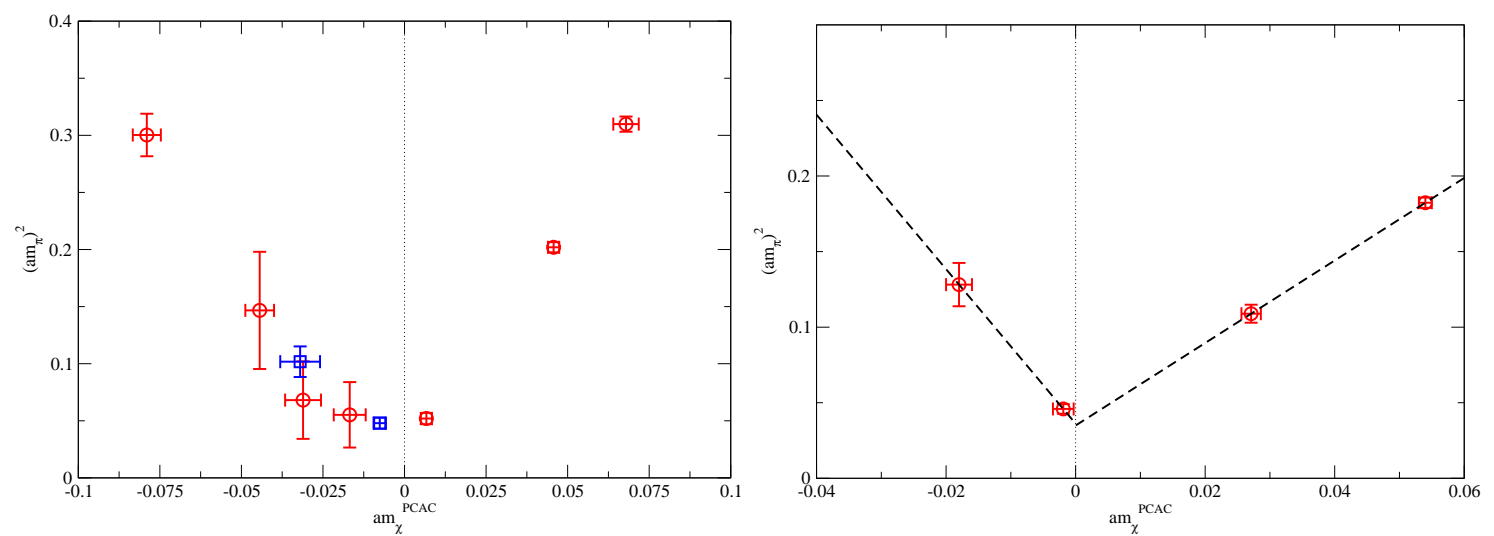

Figure 10: Pion mass squared $m_{\pi}^{2}$ vs. PCAC quark mass $m \chi^{\mathrm{PCAC}}$ from hot (red circles) and cold starts (blue squares). At $\beta=3.75, r_{0} \mu=0.020$ on a $12^{3} \cdot 24$ (left plot) and $\beta=3.90, r_{0} \mu=0.041$ on a $16^{3} \cdot 32$ lattice (right plot).

353(22) MeV for the hot start and $m_{\pi}=382(33) \mathrm{MeV}$ for the cold start, so $m_{\pi} \simeq 370 \mathrm{MeV}$ seems to be a good estimate for the minimal pion mass that can be reached at this parameter set. For additional simulations at the same $\beta$ but on a larger volume $16^{3} \cdot 32$ at $\kappa \simeq \kappa_{c}$ and $r_{0} \mu=0.039$ we obtain $m_{\pi}=407(16) \mathrm{MeV}$. Finally, at $\beta=3.90$ the pion mass we reach at $r_{0} \mu=0.041$ is $m_{\pi}=$ 453(32) MeV, while an additional simulation at $\kappa \simeq \kappa_{c}$ and $r_{0} \mu=0.020$ gives $m_{\pi}=279(37) \mathrm{MeV}$.

\section{Chiral fits}

The extension of $\chi \mathrm{PT}$ to the case of adding a twisted mass term was considered in refs. [33, $18,19,20,21,22]$. For the low-energy constants in next-to-leading order $[34,35]$ we use the notation $L_{54}=2 L_{4}+L_{5}, L_{86}=2 L_{6}+L_{8}, W_{54}=2 W_{4}+W_{5}, W_{86}=2 W_{6}+W_{8}, W=\frac{1}{2}\left(W_{86}-2 L_{86}\right)$, $\widetilde{W}=\frac{1}{2}\left(W_{54}-L_{54}\right)$ where the $L_{i}$ are the Gasser-Leutwyler coefficients and the $W_{i}$ are the corresponding coefficients of the $O\left(m_{q} a\right)$ corrections. We consider the dependence of lattice quantities on the untwisted PCAC quark mass $m_{\chi}^{P C A C}$, so we define $\chi_{P C A C}^{\prime}=2 B_{0} m_{\chi R}^{P C A C}$ where we explicitly express that $m_{\chi}^{P C A C}$ is renormalized in some prescription: $m_{\chi R}^{P C A C}=Z_{A} Z_{P}^{-1} m_{\chi}^{P C A C}$. We also define the combination containing the physical quark mass $\bar{\chi}=2 B_{0} \sqrt{\left(m_{\chi R}^{P C A C}\right)^{2}+\mu_{R}^{2}}$ with $\mu_{R}=Z_{P}^{-1} \mu$. Defining $\rho=2 W_{0} a$, where $W_{0}$ is the low-energy scale for $O(a)$ breaking (analogous to $B_{0}$ ), $F_{0}$ the pion decay constant in the chiral limit, and $\Lambda$ the renormalization scale, $\chi \mathrm{PT}$ at next-to-leading order with $O(a)$ corrections gives:

$$
\begin{aligned}
m_{\pi^{ \pm}}^{2} & =\bar{\chi}+\frac{1}{32 \pi^{2} F_{0}^{2}} \bar{\chi}^{2} \ln \frac{\bar{\chi}}{\Lambda^{2}}+\frac{8}{F_{0}^{2}}\left\{\left(-L_{54}+2 L_{86}\right) \bar{\chi}^{2}+2(W-\widetilde{W}) \rho \chi_{P C A C}^{\prime}\right\} \\
\frac{f_{\pi R}}{F_{0}} & =1-\frac{1}{16 \pi^{2} F_{0}^{2}} \bar{\chi} \ln \frac{\bar{\chi}}{\Lambda^{2}}+\frac{4}{F_{0}^{2}}\left\{L_{54} \bar{\chi}+2 \widetilde{W} \rho \frac{\chi_{P C A C}^{\prime}}{\bar{\chi}}\right\} \\
\frac{g_{\pi R}}{F_{0} B_{0}} & =1-\frac{1}{32 \pi^{2} F_{0}^{2}} \bar{\chi} \ln \frac{\bar{\chi}}{\Lambda^{2}}+\frac{4}{F_{0}^{2}}\left\{\left(-L_{54}+4 L_{86}\right) \bar{\chi}+(4 W-2 \widetilde{W}) \rho \frac{\chi_{\mathrm{PCAC}}^{\prime}}{\bar{\chi}}\right\} .
\end{aligned}
$$

We set $\Lambda=4 \pi F_{0}$. 
We performed a combined chiral fit of the quantities $m_{\pi}^{2}, f_{\pi}$ and $g_{\pi}$ for our data from the Wilson plaquette and the DBW2 gauge action (including three and two different $\beta$-values, respectively, see sec. 3 and 4 ) in order to obtain estimates for the combinations of coefficients $L_{86}, L_{54}, W, \tilde{W}$ and of the low-energy constants $B_{0}$ and $F_{0}$. In fig. 11 we show the result of this global chiral fit for the case of the pion mass, for the Wilson plaquette action (left) and the DBW2 action (right). In the former case three values of $\beta$ are included.
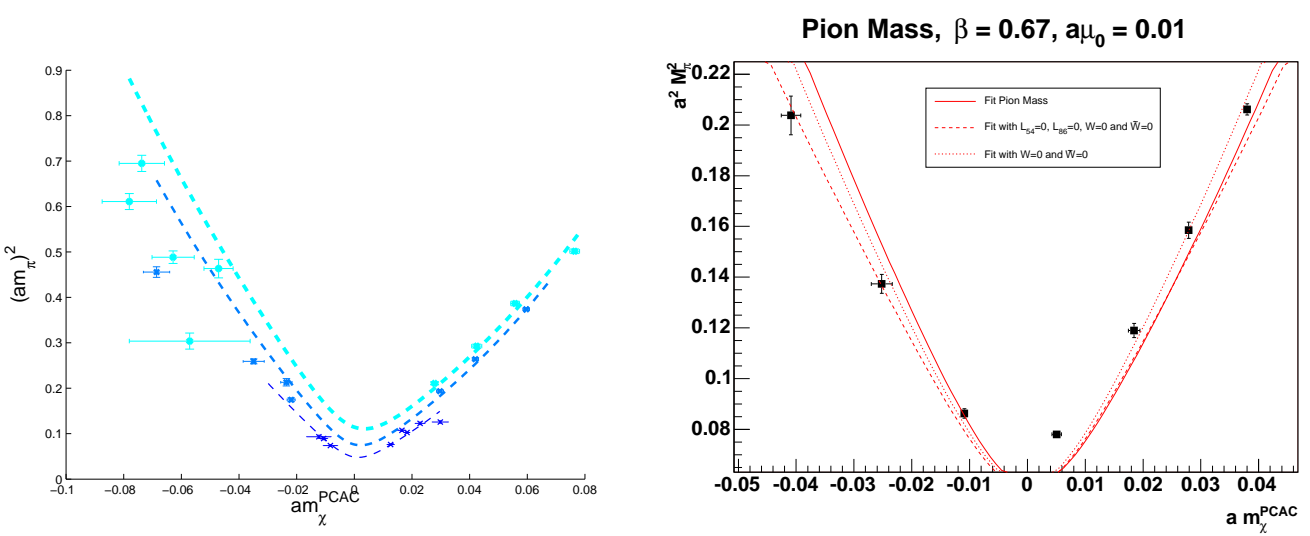

Figure 11: Chiral fit of the pion mass: Wilson plaquette action (left) and DBW2 action (right). In the Wilson case a darker blue tone corresponds to a larger value of $\beta$.

The general observation is that lattice $\chi \mathrm{PT}$ theory formulae reproduce rather well the lattice data. The $O(a)$ terms of the chiral expansion are in general not dominant in the fits, which one can infer from the fact that the associated coeffiecients fluctuate with large deviations around zero. This is probably due to the use of the PCAC quark mass which in past experiences has shown to have reduced lattice artefacts compared to the $1 / 2 \kappa$ definition of the bare quark mass. In fact fig. 2 (right panel) shows the presence of large lattice artifacts.

The use of different setups for the fits (different sets of data-points and actions, independent fit methodologies) allowed to test the stability of the results for the low-energy constants. Combining all information, we get the following ranges (here we fix the lattice scale by using the chiralextrapolated value of the Sommer scale $\left.r_{0}\right): F_{0}=70-100 \mathrm{MeV}, B_{0} / Z_{P}=3-6 \mathrm{GeV}, L_{54}=(0.8-$ $1.8) \cdot 10^{-3}, L_{86}=(0.5-1.0) \cdot 10^{-3}$. As mentioned, the coefficients of the $O(a)$ terms could not be determined.

\section{7. $N_{f}=2+1+1$ flavors of twisted mass fermions}

In this section we consider simulations of QCD in the twisted mass setup including the dynamics of the strange $(s)$ and charm (c) flavors. The $u$ and $d$ quarks, which are degenerate, are still described by the action (2.1).

\subsection{Split doublet}

The mass-splitting in the charm-strange (cs) sector is realized by adding an extra mass-term [36] 
in the action for a doublet of degenerate twisted mass fermions:

$$
S_{q}^{c s}=\sum_{x}\left\{\left(\bar{\chi}_{x}^{c s}\left[\mu_{\kappa}^{c s}+i \gamma_{5} \tau_{3} a \mu_{c s}-\tau_{1} a \mu_{\delta}\right] \chi_{x}^{c s}\right)-\frac{1}{2} \sum_{\mu= \pm 1}^{ \pm 4}\left(\bar{\chi}_{x+\hat{\mu}}^{c s} U_{x \mu}\left[r+\gamma_{\mu}\right] \chi_{x}^{c s}\right)\right\}
$$

where we use the same notation as in eq. (2.1), in particular $\mu_{\kappa}^{c s} \equiv a m_{0}^{c s}+4 r=1 / 2 \kappa_{c s}$. The new term with coefficient $\mu_{\delta}$ (the minus sign is conventional) is responsible for the splitting. This construction preserves reality and, for $\mu_{c s}^{2}>\mu_{\delta}^{2}$, positivity of the fermionic determinant. The particle content of the theory is more transparent in a different basis when $\chi_{x} \rightarrow \exp \left\{i \frac{\pi}{4} \tau_{2}\right\} \chi_{x}$, $\bar{\chi}_{x} \rightarrow \bar{\chi}_{x} \exp \left\{-i \frac{\pi}{4} \tau_{2}\right\}$, where the mass term in the untwisted direction is diagonal and the twist is in the $\tau_{1}$ flavor direction:

$$
S_{q, \text { mass }}^{c s}=\sum_{x} \bar{\chi}_{x}^{c s}\left[\mu_{\kappa}^{c s}+\tau_{3} a \mu_{\delta}+i \gamma_{5} \tau_{1} a \mu_{c s}\right] \chi_{x}^{c s}
$$

In analogy with the degenerate case the "average" quark mass of the split-doublet is given by (we neglect here for simplicity renormalization factors) $m_{c s}=\sqrt{\left(m_{0}^{c s}-m_{0 c r}^{c s}\right)^{2}+\mu_{c s}{ }^{2}}$ and the individual masses by $m_{c, s}=m_{c s} \pm \mu_{\delta}$. At full twist:

$$
m_{u}=m_{d}=\mu_{u d}, m_{c}=\mu_{c s}+\mu_{\delta}, m_{d}=\mu_{c s}-\mu_{\delta} .
$$

\subsection{Tuning to full twist}

Tuning to full twist the $N_{f}=2+1+1$ theory looks apparently more complicated because of the presence of two tunable variables $m_{0}^{u d}$ and $m_{0}^{c s}$ (or hopping parameters $\kappa_{u d}$ and $\kappa_{c s}$ ) with their respective critical values. This problem can be however circumvented in a strategy analogous to the $N_{f}=2$ case.

As we have seen previously in this contribution for the $N_{f}=2$ theory, one possible definition of the critical quark mass $m_{0 c r}\left(g_{0}, \mu\right)$ is given by the vanishing of the corresponding PCAC quark mass $m_{\chi}^{P C A C}$. Due to chirality breaking the latter gets shifted:

$$
m_{\chi}^{P C A C}=m_{0}-a^{-1} f\left(g_{0}, a m_{0}, a \mu\right),
$$

with $f$ a dimensionless function. On the basis of the symmetry of the action under parity $\times(\mu \rightarrow$ $-\mu$ ) one can show that the additive renormalization of the quark mass is even in $\mu$, and analyticity in turn implies

$$
f\left(g_{0}, a m_{0}, a \mu\right)=f\left(g_{0}, a m_{0}\right)+O\left(\mu^{2} a^{2}\right),
$$

where $f\left(g_{0}, a m_{0}\right)$ is the shift for ordinary $N_{f}=2 \mathrm{QCD}$ without twisted mass term. So the twisted mass term in the action only produces an $O(a)$ effect on the quark mass (with $g_{0}$ and $m_{0}$ held fixed):

$$
m_{\chi}^{P C A C}=m_{0}-a^{-1} f\left(g_{0}, a m_{0}\right)+O(a) .
$$

The above argument can be easily generalized to the $N_{f}=2+1+1$ theory. Here one has to make a distinction between the two sectors:

$$
\begin{aligned}
& m_{\chi(u d)}^{P C A C}=m_{0}^{u d}-a^{-1} f_{u d}\left(g_{0}, a m_{0}^{u d}, a m_{0}^{c s}, a \mu_{u d}, a \mu_{c s}, a \mu_{\delta}\right), \\
& m_{\chi(c s)}^{P C A C}=m_{0}^{c s}-a^{-1} f_{c s}\left(g_{0}, a m_{0}^{c s}, a m_{0}^{u d}, a \mu_{c s}, a \mu_{u d}, a \mu_{\delta}\right) .
\end{aligned}
$$


The functions $f_{u d}$ and $f_{c s}$ are in this case even in $\mu_{u d}, \mu_{c s}$ and $\mu_{\delta}^{\dagger}$ : similarly to $N_{f}=2$, the associated terms in the action only affect the additive renormalization of the quark mass by $O(a)$ terms. So we write:

$$
\begin{aligned}
& m_{\chi(u d)}^{P C A C}=m_{0}^{u d}-a^{-1} f\left(g_{0}, a m_{0}^{u d}, a m_{0}^{c s}\right)+O(a), \\
& m_{\chi(c s)}^{P C A C}=m_{0}^{c s}-a^{-1} f\left(g_{0}, a m_{0}^{c s}, a m_{0}^{u d}\right)+O(a),
\end{aligned}
$$

where on the r.h.s. we have now the mass-shifts for the theory without twist and mass-splitting $\left(N_{f}=2+2\right.$ QCD): here the distinction between the two sectors is immaterial. From eqs. (7.9), (7.10) it follows immediately

$$
m_{0}^{u d}=m_{0}^{c s}=m_{0} \quad \Rightarrow \quad m_{\chi(c s)}^{P C A C}=m_{\chi(u d)}^{P C A C}+O(a) .
$$

The above result suggests to tune $m_{0}^{u d}$ to its critical value where $m_{\chi(u d)}^{P C A C}=0$ keeping $m_{0}^{c s}=m_{0}^{u d}$ (or $\left.\kappa_{c s}=\kappa_{u d}\right)$ : in this situation $m_{\chi(c s)}^{P C A C}=O(a)$. Observe that since the average quark mass in the $c s$ sector is typically large, the $O(a)$ error is expected not to affect the full twist improvement in the sense of [37], while it is critical to have good tuning in the light quark sector.

\subsection{Numerical simulations}

Our strategy is to simulate the $N_{f}=2+1+1$ theory at full twist. As previously explained for a given value of $\beta$ this can be obtained by fixing $\kappa_{c s}=\kappa_{u d}=\kappa$ and tuning the PCAC quark mass in the light quark sector to zero (or equivalently by tuning the twist angle to $\pi / 2$, see sec. 4 ). At the critical value $\kappa=\kappa_{c r}, m_{\chi(u d)}^{P C C A C}=0$ by definition and $m_{\chi(c s)}^{P C A C}=O(a)$, where the $O(a)$ error in the $c s$ sector is not expected to spoil $O(a)$ improvement.

For the gauge action we choose the tree-level Symanzik improved action, which shows for $N_{f}=2$ an acceptable behavior with respect to the phase transition at small quark masses and in addition presents several more advantages in comparison with the other possible candidate DBW2, see also section 5 .

At present, we are simulating on two lattice-sizes: $12^{3} \cdot 24$ and $16^{3} \cdot 32$, our preliminary objective being the reproduction of the physical situation of previous simulation points [16] for the $N_{f}=2$ theory. This means a lattice extension $L=2 \mathrm{fm}$ and $r_{0} / a=3$ and 4 (corresponding to lattice spacings $a \simeq 0.17 \mathrm{fm}$ and $0.12 \mathrm{fm}$, respectively), lightest pion mass $m_{\pi} \simeq 400 \mathrm{MeV}\left(m_{u d} \simeq m_{s} / 3\right)$ and $300 \mathrm{MeV}\left(m_{u d} \simeq m_{s} / 5\right)$.

The $s$ and $c$ quark masses need to be tuned to their physical values. To this end we measure the kaon and $D$ meson masses and monitor the dimensionless quantities $r_{0} m_{K}$ and $r_{0} m_{D}$ which in nature assume the values $r_{0} m_{K} \simeq 1.25$ and $r_{0} m_{D} \simeq 4.7$. Due to the $\tau_{1}$ term in the action, see eq. (7.2), kaons and $D$ mesons get mixed in the twisted theory. We solve the problem by diagonalizing the two-by-two matrix of the $K$ - $D$ correlators.

The analysis of the spectrum in the $c s$ mesonic sector $\left(D_{s}\right)$ is even more awkward, again due to flavor mixing. The $\tau_{1}$ term in the action introduces indeed off-diagonal matrix elements in the

${ }^{\dagger}$ An additional symmetry in the $c s$ sector is needed for the argument, namely $\chi_{x} \rightarrow \exp \left\{i \frac{\pi}{2} \tau_{1}\right\} \chi_{x}, \bar{\chi}_{x} \rightarrow$ $\chi_{x} \exp \left\{-i \frac{\pi}{2} \tau_{1}\right\}$ composed with $\mu_{\delta} \rightarrow-\mu_{\delta}$. 
Table 3: Simulation parameters of the performed simulations. See the text for the explanations of the symbols.

\begin{tabular}{ccccccc}
\hline \hline run & $\beta$ & $\kappa$ & $a \mu_{u d}$ & $a \mu_{c s}$ & $a \mu_{\delta}$ & $N_{\text {conf }}$ \\
\hline \hline$(a)$ & 3.30 & 0.1720 & 0.01 & 0.325 & 0.275 & 550 \\
$(b)$ & 3.30 & 0.1725 & 0.01 & 0.325 & 0.275 & 920 \\
$(c)$ & 3.30 & 0.1730 & 0.01 & 0.325 & 0.275 & 900 \\
$(d)$ & 3.30 & 0.1735 & 0.01 & 0.325 & 0.275 & 730 \\
$(e)$ & 3.30 & 0.1740 & 0.01 & 0.325 & 0.275 & 590 \\
\hline \hline
\end{tabular}

Table 4: Measured quantities: the Sommer scale $r_{0} ; \rho$ meson mass $m_{\rho}$, pion mass $m_{\pi}$ and PCAC quark mass $a m_{\chi(u d)}^{P C A C}$ (light quark sector); kaon, $D$ and $D_{s}$ meson masses. For the $D_{s}$ meson only connected diagrams have been taken into account.

\begin{tabular}{cccccccc}
\hline \hline run & \multicolumn{1}{c}{$r_{0} / a$} & $a m_{\pi}$ & \multicolumn{1}{c}{$a m_{\rho}$} & $a m_{K}$ & $a m_{D}$ & $a m_{D s}$ & $a m_{\chi(u d)}^{P C A C}$ \\
\hline \hline$(a)$ & $2.23(7)(10)$ & $0.7102(38)$ & $0.8884(41)$ & $0.9551(21)$ & $1.456(10)$ & $1.6456(49)$ & $0.08795(57)$ \\
$(b)$ & $2.40(5)(5)$ & $0.6069(61)$ & $0.8075(38)$ & $0.9078(41)$ & $1.420(11)$ & $1.6385(47)$ & $0.06399(73)$ \\
$(c)$ & $3.05(3)(8)$ & $0.3463(49)$ & $0.633(13)$ & $0.7940(16)$ & $1.247(19)$ & $1.5684(59)$ & $0.01589(82)$ \\
$(d)$ & $4.11(6)(10)$ & $0.6012(88)$ & $0.981(14)$ & $0.9074(30)$ & $1.119(18)$ & $1.4074(58)$ & $-0.0711(21)$ \\
$(e)$ & $4.45(9)(20)$ & $0.808(33)$ & $1.1429(47)$ & $1.0004(17)$ & $1.311(11)$ & $1.4086(54)$ & $-0.1208(46)$ \\
\hline \hline
\end{tabular}

quark propagator which in turn produce disconnected diagrams for the $D_{s}$ hadronic correlators; denoting with $\Delta_{x y}^{f f^{\prime}}$ the quark propagator, one has $C_{D_{s}(d i s c)}\left(x_{0}, y_{0}\right)=\sum_{\vec{x}}\left\langle\operatorname{Tr}\left\{\Delta_{x x}^{c s} \gamma_{5}\right\} \operatorname{Tr}\left\{\Delta_{y y}^{s c} \gamma_{5}\right\}\right\rangle$. This disconnected term is produced by $O(a)$ flavor symmetry breaking of the twist-term, similarly to that for the neutral pion in the light quark sector, cf. the contribution [38] and ref. [39] for a discussion of this subject. (The present case is however a bit different, since the disconnected term originates from direct mixing between $c$ and $s$ quarks, while in the neutral pion case it is due to a mismatch in the sign of the twisted mass for $u$ and $d$ quarks.) In this preliminary study we measure the $D_{s}$ meson mass without taking into account the disconnected contribution.

The simulation of the $N_{f}=2+1+1$ theory is performed by a polynomial hybrid Monte Carlo algorithm (PHMC) [40]. The structure of the algorithm goes along the lines indicated in [41]. In table 3 we report the parameters of the first simulations performed. The parameters were chosen in such a way that the bare quark masses follow at full twist the proportions $m_{u}: m_{d}: m_{s}: m_{c}=1: 1$ : $5: 60$, see (7.3) (of course renormalizations can alter these proportions). The point at $\kappa=0.1730$ is the one nearest to full twist where the pion mass is minimal: here $r_{0} / a \simeq 3, m_{\pi} \simeq 400 \mathrm{MeV}$ and the $D$ meson mass is near to the physical value, but the kaon is heavier than in nature: $r_{0} m_{K} \simeq 2.4$. So the next step will be to decrease the $s$ quark mass. Simulations on a $16^{3} \cdot 32$ lattice have been also started. 
One interesting subject of investigation in perspective is of course the first order phase transition at small quark masses in this new $N_{f}=2+1+1$ setup, and in particular how it compares with previous studies with different gauge actions and/or quark flavor content $\left(N_{f}=2\right.$ and Wilson plaquette, DBW2 or tlSym gauge actions). The question arises, how the dynamics of the $s$ quark can influence the phase transition and, more in general, the low-energy features of QCD. For the moment we can only state that no signs of metastabilities were found in the present runs, which could however be just too far from the metastable region.

\section{Conclusions}

We have investigated the phase structure of lattice QCD by probing different gauge actions, adding to the standard Wilson plaquette action a term proportional to the rectangular plaquette, see eq. (2.2). By varying the coupling $c_{1}$ which multiplies the rectangular plaquette term, one can interpolate between various actions and this allows to understand in more detail the properties of the phase structure, in particular how the strength of the transition depends on the additional term and how this influences the approach to the continuum limit. For the fermion action we always used Wilson twisted mass fermions at various values of the twisted mass parameter $\mu$.

That even a small value of $c_{1}$ can already have a large impact on the phase structure is illustrated in figure 12 where we show the average plaquette value as a function of the hopping parameter $\kappa$ for three different actions, i.e. values of $c_{1}$, namely $c_{1}=0$ (Wilson), $c_{1}=-1 / 12$ (tlSym) and $c_{1}=-1.4088$ (DBW2). As one moves $\kappa$ from the negative or positive side across the

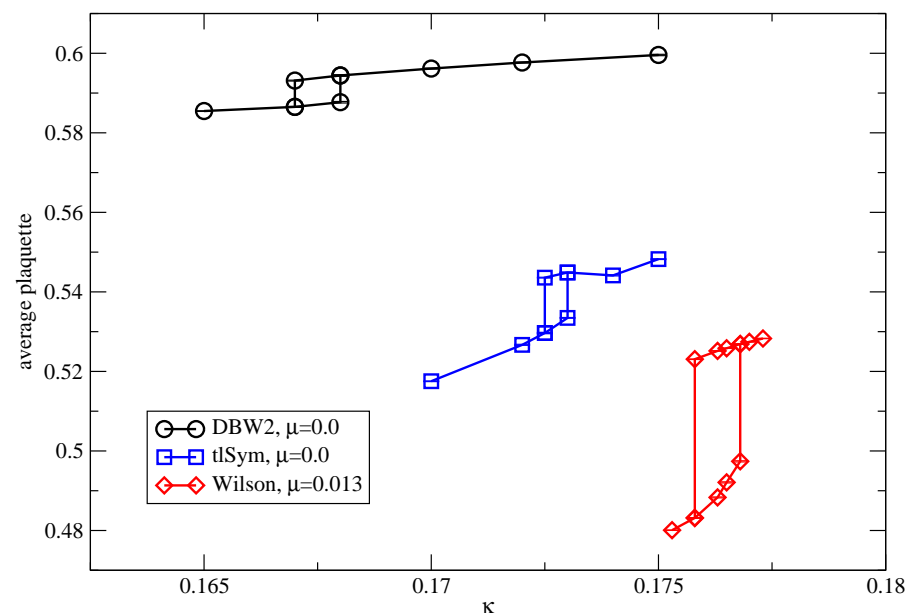

Figure 12: Hysteresis of the average plaquette value as $\kappa$ is moved across the critical point, for Wilson, tlSym and DBW2 gauge action at $a \sim 0.17 \mathrm{fm}$.

critical point, where the PCAC quark mass vanishes, a hysteresis in the average plaquette value develops of which the size and the width is an indicator of the strength of the phase transition. We observe that both the width and the size of the gap in the plaquette value decreases considerably as we switch on $c_{1}$ to $c_{1}=-1 / 12$ (tlSym action). Decreasing $c_{1}$ further down to $c_{1}=-1.4088$ (DBW2 action) still seems to reduce the size of the gap, but the effect is surprisingly small despite the large change in $c_{1}$. Note that the results in figure 12 are for a lattice spacing $a \sim 0.17 \mathrm{fm}$ that is roughly consistent for all three actions. 
However, the results for the Wilson plaquette gauge action are at non-zero twisted mass $\mu=$ 0.013, while they are at zero twisted mass for the tlSym and the DBW2 action. Since the strength of the phase transition is expected to be reduced as one switches to a non-zero twisted mass, a true comparison at $\mu=0$ would disfavor the Wilson plaquette gauge action even more. Note also that the phase transition is a generic feature of dynamical Wilson fermion simulations independent of whether a twisted mass is switched on, i.e. the transition also occurs for standard Wilson fermion simulations. Another feature of the first order phase transition is that its strength weakens rapidly when the lattice spacing is made finer. This is illustrated in fig. 2 for the case of the Wilson plaquette gauge action.

A satisfactory setup for dynamical simulations with, say, $N_{f}=2$ flavors of quarks would be to reach pion masses of about $250-300 \mathrm{MeV}$ and a box size of $L>2 \mathrm{fm}$. At the same time, one should stay at full twist to realize $\mathrm{O}(\mathrm{a})$-improvement. From our preliminary results we find that for the tlSym action this can be achieved with a reasonable computer time at $\beta=3.9$ on $L / a=20$ lattices. For smaller values of $\beta$ we find that at pion masses of about $400 \mathrm{MeV}$ large fluctuations appear, although no clear signs of metastabilities are visible. Although for the DBW2 action the situation might be somewhat better, the advantages of the t1Sym action such as good convergence of perturbation theory and small scaling violations as found quenched, lead us to decide on the tlSym gauge action as the action of choice.

For our present results from simulations using the Wilson plaquette and the DBW2 gauge actions we found that $\chi \mathrm{PT}$ describes the data rather well and allows to extract a number of lowenergy constants of the effective chiral Lagrangian. We have also seen that scaling violations of both actions are not very large when we compared physical results at different values of the lattice spacing.

Very good news is that simulations with $N_{f}=2+1+1$ flavors of dynamical quarks are perfectly feasible with the twisted mass formulation of lattice QCD. Our first and preliminary results are very promising. The (PHMC) algorithm works nicely and, even more important, it seems that the tuning in this physically more realistic setup is quite manageable. Taken this fact together with the new developments of fast versions of the HMC algorithm to simulate dynamical quarks, it seems natural to start now realistic simulations with twisted mass fermions.

\section{Acknowledgments}

We thank H. Perlt for providing us with the perturbative estimates of the renormalization constants of the quark bilinears for the DBW2 gauge action. We thank R. Frezzotti, G. C. Rossi and S. Sharpe for many useful discussions. The computer centers at NIC/DESY Zeuthen, NIC at Forschungszentrum Jülich and HLRN provided the necessary technical help and computer resources. We are indebted to R. Hoffmann and J. Rolf for leaving us a MatLab program to check our fits. This work was supported by the DFG Sonderforschungsbereich/Transregio SFB/TR9-03.

\section{References}

[1] I. Wetzorke et. al., Dynamical Wilson twisted mass fermions: A scaling analysis, POS (LAT2005) 074. 
[2] N. Ukita et. al., Scaling test of dynamical wilson twisted mass fermions with DBW2 gauge action, POS (LAT2005) 037.

[3] U. Wenger et. al., Lattice QCD with $N_{f}=2$ light Wilson fermions: the phase structure and scaling, POS (LAT2005) 044.

[4] K. Symanzik, Continuum limit and improved action in lattice theories. 1. principles and phi**4 theory, Nucl. Phys. B226 (1983) 187.

[5] K. Symanzik, Continuum limit and improved action in lattice theories. 2. o(n) nonlinear sigma model in perturbation theory, Nucl. Phys. B226 (1983) 205.

[6] S. R. Sharpe and J. Singleton, R., Spontaneous flavor and parity breaking with Wilson fermions, Phys. Rev. D58 (1998) 074501 [hep-lat/9804028].

[7] F. Farchioni et. al., Twisted mass quarks and the phase structure of lattice QCD, Eur. Phys. J. C39 (2005) 421-433 [hep-lat/ 0406039$].$

[8] F. Farchioni et. al., Exploring the phase structure of lattice QCD with twisted mass quarks, Nucl. Phys. Proc. Suppl. 140 (2005) 240-245 [hep-lat/ 0409098$].$

[9] R. Frezzotti, P. A. Grassi, S. Sint and P. Weisz, A local formulation of lattice QCD without unphysical fermion zero modes, Nucl. Phys. Proc. Suppl. 83 (2000) 941-946 [hep-lat/9909003].

[10] R. Frezzotti, Wilson fermions with chirally twisted mass, Nucl. Phys. Proc. Suppl. 119 (2003) 140-148 [hep-lat/0210007].

[11] R. Frezzotti, Twisted mass lattice qcd, Nucl. Phys. Proc. Suppl. 140 (2005) 134-140 [hep-lat/0409138].

[12] A. Shindler, Twisted mass lattice qcd: Recent developments and results, POS (LAT2005) 014.

[13] S. Aoki, New phase structure for lattice QCD with Wilson fermions, Phys. Rev. D30 (1984) 2653.

[14] E.-M. Ilgenfritz, W. Kerler, M. Müller-Preußker, A. Sternbeck and H. Stüben, A numerical reinvestigation of the Aoki phase with $N(f)=2$ Wilson fermions at zero temperature, Phys. Rev. D69 (2004) 074511 [hep-lat/0309057].

[15] A. Sternbeck, E.-M. Ilgenfritz, W. Kerler, M. Müller-Preußker and H. Stüben, The Aoki phase for N(f) = 2 Wilson fermions revisited, Nucl. Phys. Proc. Suppl. 129 (2004) 898-900 [hep-lat/ 0309059 ].

[16] F. Farchioni et. al., The phase structure of lattice QCD with Wilson quarks and renormalization group improved gluons, Eur. Phys. J. C42 (2005) 73-87 [hep-lat/ 0410031 ].

[17] F. Farchioni et. al., Lattice spacing dependence of the first order phase transition for dynamical twisted mass fermions, accepted for publ. in Phys. Lett. B [hep-lat/0506025].

[18] G. Münster, On the phase structure of twisted mass lattice QCD, JHEP 09 (2004) 035 [hep-lat/0407006].

[19] L. Scorzato, Pion mass splitting and phase structure in twisted mass QCD, Eur. Phys. J. C37 (2004) 445-455 [hep-lat/0407023].

[20] S. R. Sharpe and J. M. S. Wu, Twisted mass chiral perturbation theory at next-to-leading order, Phys. Rev. D71 (2005) 074501 [hep-lat/ 0411021$].$

[21] S. R. Sharpe and J. M. S. Wu, The phase diagram of twisted mass lattice QCD, Phys. Rev. D70 (2004) 094029 [hep-lat/0407025]. 
[22] S. Aoki and O. Bär, Twisted-mass $Q C D, O(a)$ improvement and Wilson chiral perturbation theory, Phys. Rev. D70 (2004) 116011 [hep-lat/ 0409006 ].

[23] T. Takaishi, Heavy quark potential and effective actions on blocked configurations, Phys. Rev. D54 (1996) 1050-1053.

[24] P. Weisz, Continuum limit improved lattice action for pure yang-mills theory. 1, Nucl. Phys. B212 (1983) 1.

[25] R. Sommer, A new way to set the energy scale in lattice gauge theories and its applications to the static force and alpha-s in SU(2) Yang-Mills theory, Nucl. Phys. B411 (1994) 839-854 [hep-lat/9310022].

[26] C. Urbach, K. Jansen, A. Shindler and U. Wenger, HMC algorithm with multiple time scale integration and mass preconditioning, accepted for publ. in Comp. Phys. Commun. [hep-lat/0506011].

[27] C. Urbach et. al., HMC algorithm with multiple time scale integration and mass preconditioning, POS (LAT2005) 118.

[28] $\chi_{\mathbf{E}} \mathbf{E}$ Collaboration, K. Jansen, M. Papinutto, A. Shindler, C. Urbach and I. Wetzorke, Quenched scaling of Wilson twisted mass fermions, accepted for publ. in JHEP [hep-lat/0507010].

[29] R. Frezzotti and S. Sint, Some remarks on O(a) improved twisted mass QCD, Nucl. Phys. Proc. Suppl. 106 (2002) 814-816 [hep-lat/0110140].

[30] $\chi \mathbf{E}$ Collaboration, K. Jansen, A. Shindler, C. Urbach and I. Wetzorke, Scaling test for Wilson twisted mass QCD, Phys. Lett. B586 (2004) 432-438 [hep-lat / 0312013 ].

[31] L. Maiani and G. Martinelli, Current algebra and quark masses from a monte carlo simulation with wilson fermions, Phys. Lett. B178 (1986) 265.

[32] QCDSF Collaboration, H. Perlt, private communication.

[33] G. Münster and C. Schmidt, Chiral perturbation theory for lattice qcd with a twisted mass term, Europhys. Lett. 66 (2004) 652-656 [hep-lat/0311032].

[34] G. Rupak and N. Shoresh, Chiral perturbation theory for the Wilson lattice action, Phys. Rev. D66 (2002) 054503 [hep-lat/0201019].

[35] O. Bär, G. Rupak and N. Shoresh, Chiral perturbation theory for lattice qcd at o(alpha**2), Nucl. Phys. Proc. Suppl. 129 (2004) 185-187 [hep-lat/0309033].

[36] R. Frezzotti and G. C. Rossi, Twisted-mass lattice qcd with mass non-degenerate quarks, Nucl. Phys. Proc. Suppl. 128 (2004) 193-202 [hep-lat/0311008].

[37] R. Frezzotti and G. C. Rossi, Chirally improving Wilson fermions. I: O(a) improvement, JHEP 08 (2004) 007 [hep-lat/0306014].

[38] F. Farchioni et. al., Twisted mass fermions: neutral pion masses from disconnected contributions, POS (LAT2005) 033.

[39] XLF Collaboration, K. Jansen et. al., Flavour breaking effects of wilson twisted mass fermions, accepted for publ. in Phys. Lett. B [hep-lat/0507032].

[40] R. Frezzotti and K. Jansen, A polynomial hybrid monte carlo algorithm, Phys. Lett. B402 (1997) 328-334 [hep-lat/9702016].

[41] I. Montvay and E. Scholz, Updating algorithms with multi-step stochastic correction, Phys. Lett. B623 (2005) 73-79 [hep-lat/ 0506006 ]. 